\title{
La démobilisation étudiante au Mexique : le double visage de la répression (juillet-décembre 1968)
}

Guadalupe Olivier-Téllez, Sergio Tamayo Flores-Alatorre et Michael Voegtli

\section{Q OpenEdition}

Édition électronique

URL : http://journals.openedition.org/ejts/4819

DOI : $10.4000 /$ ejts.4819

ISSN : $1773-0546$

Éditeur

EJTS

\section{Référence électronique}

Guadalupe Olivier-Téllez, Sergio Tamayo Flores-Alatorre et Michael Voegtli, « La démobilisation étudiante au Mexique : le double visage de la répression (juillet-décembre 1968) », European Journal of Turkish Studies [En ligne], 17 | 2013, mis en ligne le 01 avril 2014, consulté le 16 février 2020. URL: http://journals.openedition.org/ejts/4819; DOI : 10.4000/ejts.4819

Ce document a été généré automatiquement le 16 février 2020.

(c) Some rights reserved / Creative Commons license 


\title{
La démobilisation étudiante au Mexique : le double visage de la répression (juillet-décembre 1968)
}

\author{
Guadalupe Olivier-Téllez, Sergio Tamayo Flores-Alatorre et Michael Voegtli
}

\section{NOTE DE L'AUTEUR}

Les auteurs remercient les réviseurs anonymes pour leurs remarques et commentaires sur des versions antérieures de ce texte.

« Vaincra l'indignation ou la peur » (Marcelino Perelló Vals, étudiant activiste) ${ }^{1}$

1 Indignation et peur sont deux émotions fréquemment mentionnées par les intégrant-es du mouvement étudiant de 1968 au Mexique, mouvement violemment réprimé par le régime autoritaire. L'indignation initiale qui déclenche la mobilisation à la suite des premières actions de répression des étudiant-e-s par la police et l'armée laisse place à la peur au fur et à mesure que la technologie de la répression s'accroît. Présente dès l'origine du mouvement, la répression n'est ainsi pas suffisante pour comprendre la démobilisation étudiante. Elle ne prend sens que lorsqu'elle est liée aux risques de la participation et aux affects dans une configuration spécifique.

2 Cet article étudie la mobilisation et la démobilisation étudiante dans le cas du mouvement de 1968 dans la ville de Mexico. Considérant la période qui va du 23 juillet au 6 décembre 1968, date marquée par la dissolution formelle du Conseil national de grève (Consejo Nacional de Huelga, $\mathrm{CNH}$ ), nous privilégions l'analyse de deux dimensions $\mathrm{du}$ processus de mobilisation/démobilisation. D'une part, nous nous intéressons à la dimension organisationnelle qui en explique le déclin en le liant aux transformations $\mathrm{du}$ contexte politique, en particulier à la répression du régime autoritaire qui pèse sur les coûts et les risques individuels de la participation politique. Étudier la dynamique interne du mouvement permet ainsi de s'intéresser aux 
conditions de la poursuite de l'engagement militant en liant la participation politique à la configuration sociopolitique qui la rend possible ou la limite. D'autre part, l'examen des témoignages d'activistes du 68 mexicain $^{2}$ nous permet de mettre au jour certains mécanismes de la démobilisation et notamment le rôle des émotions. La lutte des étudiant-e-s au Mexique constitue un cas particulièrement intéressant de mobilisation et démobilisation politique. Située dans un contexte de forte contestation au niveau international, elle tire son origine de différentes causes. Nous insisterons ici sur deux niveaux.

3 Premièrement, il s'agit de se pencher sur le contexte lié à une configuration politique et socio-éducative nationale. Comme nous le verrons dans la première partie de cet article, la situation politique dominée par un système de parti unique qui ne donne pas la parole aux oppositions coexiste avec des demandes d'ouverture et de libéralisation de plusieurs secteurs. Les revendications pour plus de liberté d'opinion (libération des prisonniers politiques y comprise), de liberté de presse et pour une ouverture et démocratisation de l'accès à l'éducation se situent dans un processus plus ample de mobilisation initié plusieurs années auparavant et culminant en août 1968.

Deuxièmement, la dynamique de cette mobilisation d'une durée de sept mois renvoie aux réseaux organisationnels et à l'échange de coups entre activistes d'un côté et autorités politiques et forces de maintien de l'ordre (police et armée) de l'autre, dans une situation de couverture médiatique défavorable au mouvement. Nous verrons dans les quatre parties suivantes que la répression constitue dans un premier temps une incitation à la mobilisation et un élément de configuration du mouvement (jusqu'à la fin du mois d'août); mais à partir du moment où elle s'intensifie, elle favorise le démantèlement organisationnel du mouvement et le désengagement ${ }^{3}$.

5 La démobilisation politique a fait l'objet de nombreuses analyses, que ce soit pour en saisir la dimension collective, c'est-à-dire la fin d'une ample mobilisation sociale ou d'un mouvement, ou pour comprendre ses aspects individuels, en cernant les conditions du désengagement militant (pour une synthèse, cf. Fillieule 2013). Sans prétendre nous inscrire dans la lignée des analyses étudiant les conséquences biographiques de l'engagement (Gottraux 1997, McAdam 1999, Fillieule 2005, Leclercq et Pagis 2011), nous proposons ici d'adopter une perspective processuelle de l'action protestataire qui prenne en compte les dimensions de la configuration sociopolitique favorisant l'évolution de la mobilisation et son déclin, ainsi que les transformations organisationnelles et individuelles au cours de ce processus. Comme l'indique McAdam (1982: 48) : «Insérées entre l'opportunité et l'action, il y a les personnes et les significations subjectives qu'elles attribuent à leurs situations». Cet aspect est crucial pour comprendre le passage de la mobilisation à la démobilisation.

6 Dans ce processus, il est indispensable d'analyser le rôle de la répression, dans la mesure où plusieurs analyses soulignent qu'elle peut autant contribuer à affermir l'action protestataire qu'à l'affaiblir, selon l'étape à laquelle se trouve la mobilisation (Brockett 1993 et 2005; pour une synthèse, cf. Combes et Fillieule 2011). Ainsi, la répression ne permettant pas en elle-même d'expliquer le succès ou l'échec d'une mobilisation ; il faut dès lors saisir la dimension subjective de la participation politique dans la dynamique de la lutte.

7 Prendre en compte la dimension subjective consiste à indiquer que chaque mobilisation a ses coûts et risques, qui sont autant de conditions de poursuite de l'action. Nous nous appuyons sur les travaux de Wiltfang et McAdam (1991), selon lesquels les coûts sont 
définis comme "la dépense de temps, d'argent et d'énergie nécessaire à une personne engagée dans n'importe quelle forme d'activisme». Les risques, quant à eux, se rapportent aux «dangers anticipés - légaux, sociaux, physiques, financiers, etc. - de l'engagement dans une activité de mouvement » (McAdam, 1986:67). Le coût est tout ce qui est sacrifié, passé, dépensé, perdu ou vécu "négativement " (par exemple la souffrance, la fatigue, etc.) par les activistes au cours de leur participation aux activités du mouvement. Le risque, de l'autre côté, fait référence à l'anticipation ou à l'attente subjective de la part des activistes d'un coût qui pourrait être le résultat de leur participation à un mouvement (par exemple être arrêté, payer une amende, être battu, torturé ou tué).

«Les coûts sont sous le contrôle personnel de l'activiste; les risques, en tant que coûts futurs, ne dépendent pas seulement des propres actions de l'activiste, mais

des réponses des autres aux actions de l'activiste » (Wiltfang et McAdam 1991 : 989).

Cette définition insiste sur les dimensions objectives et subjectives de la participation politique et sur leurs transformations en fonction de comment s'articulent certaines oppositions et alliances. Loin de reprendre à leur compte une perspective telle que celle du choix rationnel, les auteurs soulignent l'importance de la relation entre les autorités politiques et les acteurs engagés dans le mouvement ; ils signalent que les changements de la dynamique de lutte influent sur les opportunités de poursuivre l'engagement, dans la mesure où les activistes réélaborent sans cesse leurs perceptions des risques.

Sur l'ensemble du processus, le poids des émotions se révèle dès lors déterminant, comme l'ont montré dans d'autres contextes plusieurs analyses sur l'action collective (notamment Jasper 1998; Goodwin, Jasper and Polletta 2004: 414; Traïni et Siméant 2009; Broqua et Fillieule 2009 ; pour une synthèse : Sommier 2010) et comme l'indique Récappé (2008) dans son analyse des ressorts de la mobilisation du mouvement étudiant mexicain. Selon Sommier (2010), la "dimension affectuelle», rassemblant sentiments et émotions, se manifeste à trois niveaux: elle est d'abord visible dans la phase de la mobilisation, lorsqu'une série d'événements répressifs générant de l'indignation contribuent à l'engagement individuel et au développement d'une large coalition; ensuite, elle est constamment perceptible au niveau de l'«économie affectuelle du groupe", que ce soit dans les "liens émotionnels" (Récappé 2008: 200) qui cimentent l'action protestataire, en particulier par l'intermédiaire du travail quotidien dans les comités et brigades, ou encore dans le maintien de la loyauté par le recours à la figure des martyrs - prisonniers politiques et morts - lors de la phase de démobilisation. Enfin, cette démobilisation ne peut être cernée sans prendre en compte les "affects négatifs ou dysphoriques" (Sommier $2010: 201$ ) et en premier lieu la peur.

10 Ainsi, mettre l'accent sur les risques de la participation en les liant aux affects dans un contexte de répression accrue permet largement d'expliquer le processus de mobilisation et de démobilisation étudiante lors du 68 mexicain et, pour cette dernière, les moments où commencent à prédominer les motifs du désengagement basés sur le vocabulaire de la peur.

11 Pour le démontrer, nous avons établi une périodisation particulière liée à la démobilisation, en identifiant quatre phases. La première, très courte, va du 23 au 30 juillet : elle débute avec la protestation étudiante à la suite de la répression par la police de lycéen-ne-s impliqué-e-s dans une bataille de rue. L'origine du mouvement est aussi le moment où commence à être orchestrée par le gouvernement une technologie 
de la répression dont l'efficacité sera visible à plus long terme. La seconde phase, du 31 juillet au 28 août, est une période qui reflète le développement d'un mouvement qui se structure au niveau de son organisation et génère des répertoires d'action d'une efficacité et d'un impact importants, formant de denses réseaux d'alliés dont les liens permettent l'alignement de cadres d'interprétation de la mobilisation. La troisième phase, du 29 août au 2 octobre, est caractérisée par l'usage insistant et ouvert de la technologie de la répression de l'État, cherchant par tous les moyens à briser les liens entre le mouvement et la société mexicaine, ébranlant les effets positifs des répertoires de mobilisation, isolant le mouvement, minant les sources de sa force sociale. La démobilisation en termes génériques commence à affaiblir le mouvement. La quatrième et dernière phase va du 3 octobre au 6 décembre, jour où cesse officiellement la grève étudiante. Il s'agit d'une étape de désagrégation générale des protagonistes, conséquence de l'emploi brutal de la technologie de la répression et d'un recours systématique aux agressions, comme l'occupation par l'armée des installations universitaires et du massacre du 2 octobre, visant particulièrement les représentants du mouvement mais aussi l'ensemble des personnes réunies dans les espaces publics. Avant d'aborder ces phases, il convient de clarifier les conditions initiales qui contribuent à l'action protestataire, en cernant les modalités de constitution du mouvement, les réseaux organisationnels à son origine et l'actualisation des répertoires d'action hérités de la longue trajectoire de lutte étudiante durant la première moitié du $\mathrm{XX}^{\mathrm{e}}$ siècle.

\section{Les origines du mouvement}

Les réseaux organisationnels peuvent favoriser la production et la circulation de ressources affectives, cognitives et symboliques (Fernandez et McAdam 1988, Snow et McAdam 2000, Diani 2004) et influencent aussi bien la configuration d'alliances possibles que les moyens et les objectifs de la lutte. La compréhension du mode de constitution de ces réseaux permet, en outre, de mettre en partie au jour les mécanismes de la démobilisation à partir du moment où se modifient l'équilibre et le soutien des organisations qui les composent.

La lutte étudiante au Mexique s'inscrit dans la vague contestataire de 1968, cette année constituant une étape de transition structurelle. La succession d'événements qui surviennent durant les années 1960 prend corps dans les soulèvements pour la justice sociale menés à l'ombre de la Guerre froide. La mort d'Ernesto Che Guevara en 1967 marque la naissance du mythe révolutionnaire et l'effervescence des positions de la gauche. Le Mai français montre aux étudiant-e-s mexicain-e-s mobilisé-e-s l'exemple d'une jeunesse radicalisée, intellectualisée et politisée. La révolte étudiante allemande, le printemps de Prague, la contestation à la guerre du Vietnam, le mouvement hippie, la théologie latino-américaine de la libération influencée par le marxisme théorique ou encore la grève de l'Association nationale des éducateurs salvadoriens (ANDES) sont autant d'exemples de luttes de libération.

14 Les dernières années de la décennie sont marquées par la rébellion : Hobsbawn (2000) s'y réfère davantage comme une porte qui se referme, la fin de la révolution de l'ancien monde, mais en même temps, dans le cas de la plupart des pays d'Amérique latine il s'agit d'années marquées par des transformations importantes, de luttes quasi permanentes. Au Mexique, culmine au cours de ces années le modèle de développement 
économique qui s'était auparavant imposé comme projet dominant: substitution des importations, développement et soutien aux entreprises nationales, essor de l'Étatprovidence. L'année 1968 constitue une transition en germe depuis plusieurs années, un nouvel horizon de modernité qui donnera forme à ce que seront les grands changements des sphères politique, sociale et culturelle du pays (Tamayo 1998).

Au cours des années 1960, la citoyenneté signifie un ordre social sous le contrôle étroit du pouvoir gouvernemental du président Gustavo Díaz Ordaz (Tamayo 1998). Ce modèle a émergé très progressivement au cours des années 1940, décennie au cours de laquelle l'État mexicain contribue à faire des secteurs intermédiaires sa nouvelle base sociale, impliquant un renouvellement des formes de domination. Les projets populaires qui caractérisaient la période de gouvernement de Lázaro Cárdenas dans une perspective socialiste sont abandonnés.

Diverses mobilisations se développent en réaction : le mouvement des cheminots et des étudiants des écoles de formation d'enseignants de 1958 à 1959, puis d'autres davantage liées aux mouvements ouvrier et paysan. Dans un contexte d'expansion des classes moyennes qui favorise un développement accéléré des zones urbaines, on assiste à une forte polarisation sociale. Au niveau éducatif, l'éducation supérieure devient plus massive et la sphère du travail se restructure exigeant désormais une formation plus poussée, sans toutefois pouvoir absorber l'ensemble des personnes issues de la formation supérieure. Martínez Della Rocca (2010) souligne le décalage entre le processus de massification des universités et l'offre limitée d'emploi dans un contexte de précarité salariale.

17 Les données suivantes de l'Université nationale autonome du Mexique (UNAM), l'établissement d'enseignement supérieur numériquement le plus important du pays jusqu'à nos jours, au cœur du conflit étudiant en 1968, permettent d'illustrer la demande croissante dans l'éducation supérieure (tableau 1) :

\begin{tabular}{|l|l|}
\hline \multicolumn{2}{|c|}{ Tableau 1. Evolution du nombre d'étudiant-e-s à l'UNAM (1950-1980) } \\
\hline Année & Nombre d'étudiant-e-s inscrit-e-s (en milliers) \\
\hline 1950 & 29,9 \\
\hline 1960 & 77,1 \\
\hline 1970 & 271,3 \\
\hline 1980 & 825,5 \\
\hline
\end{tabular}

Source : tableau élaboré à partir de données de Martínez Della Roca (2010)

Au cours des années 1960, le mécontentement des étudiant-e-s par rapport à leur avenir professionnel est clairement visible. Ce facteur contribue à la politisation des établissements d'enseignement supérieur; les critiques émergent précisément à l'intérieur des groupes sociaux de classe moyenne historiquement perçus comme étant loyaux à l'État. La prospérité liée au « miracle mexicain » depuis les années 1940 s'étiole rapidement et laisse place à une série d'expressions alternatives et de résistances dans toutes les sphères sociales. 
19 L'ère de la contre-culture imprègne différentes universités et facultés en questionnant les principes artistiques, familiaux et sociétaux dominants. Le conflit s'exacerbe au début des années 1960 et se poursuit durant toute la décennie avec des conflits dans les universités du centre et du sud du pays : Guerrero, Puebla, Michoacán et Mexico ; à la fin de la décennie, le conflit s'étend dans les États de Sinaloa et de Durango (nordouest). Parmi ces luttes, on peut mentionner celles des professeurs du Syndicat national des travailleurs dans l'éducation (SNTE) et de l'École nationale d'agriculture Chapingo qui demandent la fin de la répression et la libération des prisonniers politiques.

Dans ce cadre nous observons deux processus: le premier renvoie à la perte de légitimité de l'État face à l'université ; le second à la croissante politisation à l'intérieur de l'université. Les différentes mobilisations étudiantes, par exemple en soutien à la révolution cubaine et contre la guerre du Vietnam, reflètent les aspirations à un monde différent. À l'intérieur de l'UNAM, des groupes de gauche sont créés qui affrontent le contexte de crise universitaire, en particulier les actions répressives des autorités académiques pour empêcher les meetings et assemblées, tout en participant aussi aux luttes sociales à l'extérieur de l'université (Martínez Della Rocca 2010). Ainsi le 68 mexicain n'est pas une explosion isolée mais le résultat de l'accumulation d'un ensemble de manifestations (Récappé 2008, chap. 5 et 6) qui débutent dès la première moitié du XX siècle et qui prennent de l'ampleur à la fin des années 1950. Entre 1963 et 1968, on compte au moins 53 révoltes étudiantes (Cruz Flores 2011).

On peut dès lors affirmer, d'une part, que les événements de 1968 ont été précédés au cours des décennies antérieures par au moins une centaine de mobilisations étudiantes, certaines étroitement liées au contexte éducatif, d'autres d'ordre politique et social plus général. D'autre part, des organisations étudiantes sont créées et développées dans tout le pays (tableau 2). Certaines organisations, en particulier la Fédération nationale des étudiants de l'enseignement technique (FNET) sont très proches du gouvernement et jouent le rôle de "démobilisateurs internes». D'autres, à l'inverse, sont les principaux détracteurs de l'autoritarisme du gouvernement et luttent pour une véritable indépendance par rapport à l'État et en faveur de la démocratisation du pays.

\begin{tabular}{|c|c|}
\hline \multicolumn{2}{|c|}{ Tableau 2. Organisations étudiantes au Mexique (1920-1967) } \\
\hline Nom et année de création & Caractéristiques générales \\
\hline $\begin{array}{l}\text { Federación de Estudiantes del } \\
\text { Distrito Federal-Fédération des } \\
\text { étudiants du District fédéral } \\
\text { (FEDF). } 1920\end{array}$ & $\begin{array}{l}\text { Première organisation étudiante enregistrée. Sa lutte principale, } \\
\text { en 1929, est en faveur de l'autonomie universitaire. }\end{array}$ \\
\hline $\begin{array}{lr}\text { Confederación } & \text { Nacional de } \\
\text { Estudiantes } & \text { - Confédération } \\
\text { nationale d'étudiants (CNE). } 1924\end{array}$ & $\begin{array}{l}\text { Promeut la mise en place de normes de participation } \\
\text { démocratique, l'acceptation en son sein d'expressions politiques } \\
\text { diverses et la prise de décision consensuelle. }\end{array}$ \\
\hline $\begin{array}{l}\text { Federación de Estudiantes } \\
\text { Universitarios } \quad \text { - Fédération } \\
\text { d'étudiants universitaires (FEU). } \\
1933\end{array}$ & Libérale et indépendante de l'État. Fonctionne jusqu'en 1948. \\
\hline
\end{tabular}




\begin{tabular}{|c|c|}
\hline $\begin{array}{lr}\text { Federación } & \text { Nacional de } \\
\text { Estudiantes } & \text { Técnicos } \\
\text { Fédération } & \text { nationale } \\
\text { d'étudiants de l'enseignement } \\
\text { technique (FNET). } 1936\end{array}$ & $\begin{array}{l}\text { Réunit les écoles supérieures techniques, avec à leur tête } \\
\text { l'Institut polytechnique national (IPN). Liée au Parti de la } \\
\text { révolution mexicaine (PRM), puis au Parti révolutionnaire } \\
\text { institutionnel (PRI). }\end{array}$ \\
\hline $\begin{array}{l}\text { Confederación de Jóvenes } \\
\text { Mexicanos - Confédération des } \\
\text { jeunes Mexicains (CJM). } 1938\end{array}$ & $\begin{array}{l}\text { Émerge de la Confédération des étudiants socialistes du Mexique } \\
\text { (CESM) et des Jeunesses socialistes. Participe au renforcement } \\
\text { du corporatisme étudiant sous le parti hégémonique. }\end{array}$ \\
\hline $\begin{array}{lr}\text { Federación } & \text { Nacional de } \\
\text { Estudiantes } & \text { Campesinos } \\
\text { Socialistas } & \text { - Fédération } \\
\text { nationale des étudiants paysans } \\
\text { socialistes (FNECS). } 1938\end{array}$ & $\begin{array}{l}\text { Rassemble } 29 \text { écoles normales rurales de tout le pays. Liée au } \\
\text { PRM. }\end{array}$ \\
\hline $\begin{array}{l}\text { Federación de Estudiantes de } \\
\text { Occidente (FEO) de la } \\
\text { Universidad de Guadalajara }- \\
\text { Fédération des étudiants de } \\
\text { l'Ouest (université } \\
\text { Guadalajara). } 1938\end{array}$ & Liée a \\
\hline $\begin{array}{l}\text { Consejo de Estudiantes Técnicos } \\
\text { en defensa del IPN - Conseil des } \\
\text { étudiants de l'enseignement } \\
\text { technique en défense de l'IPN } \\
\text { (CETDIPN). } 1956\end{array}$ & $\begin{array}{l}\text { Rassemble trois délégués de chaque école supérieure } \\
\text { d'enseignement technique et un membre de la FNET. Liée au } \\
\text { PRI. }\end{array}$ \\
\hline $\begin{array}{l}\text { Central Nacional de Estudiantes } \\
\text { Democráticos } \quad \text { - Centrale } \\
\text { nationale des étudiants } \\
\text { démocratiques (CNED). } 1963\end{array}$ & $\begin{array}{l}\text { Émergence de la désarticulation de différentes organisations } \\
\text { étudiantes à la suite des processus de démobilisation orchestrés } \\
\text { par des organismes étudiants liés au PRI. Les objectifs sont :1) la } \\
\text { consolidation d'une organisation indépendante de l'appareil } \\
\text { officiel du gouvernement ; } 2 \text { ) la lutte pour ses revendications et } \\
\text { plus largement pour une transformation politique du pays. }\end{array}$ \\
\hline $\begin{array}{l}\text { Consejo Estudiantil Universitario } \\
\text { - Conseil étudiant universitaire } \\
\text { (CEU). } 1966\end{array}$ & $\begin{array}{l}\text { Produit de la grève de l'UNAM de 1966. La perspective } \\
\text { démocratico-organisationnelle est reprise dans les principes de } \\
\text { base de la Confédération nationale des étudiants de } 1929 \text { et des } \\
\text { Comités de lutte de l'éducation populaire de } 1936 \text {. }\end{array}$ \\
\hline
\end{tabular}

Source : synthèse élaborée par les auteurs.

En dépit du fait que le gouvernement s'appuie sur certains groupes pour formuler ses stratégies d'intervention dans le domaine du contrôle éducatif, d'autres organisations parviennent à demeurer indépendantes et adoptent un fonctionnement démocratique. On verra dans le cas du CNH de 1968 que cet élément constitue un enjeu central de la lutte.

23 Ces dimensions contextuelles et organisationnelles montrent ainsi que, contrairement à la vision spontanéiste de la mobilisation politique, les luttes étudiantes de 1968 s'inscrivent dans une longue trajectoire de mobilisations politiques et d'oppositions à 
l'intérieur même du mouvement étudiant entre organisations corporatives du régime du PRI et groupes de tendance démocratique. Et, comme nous allons le voir à présent, lorsqu'un premier conflit éclate en juillet 1968, c'est précisément la légitimité de la FNET, alignée au gouvernement, à représenter les attentes démocratiques d'une partie des étudiant-e-s, qui va être remise en question.

\section{$1^{\text {re }}$ phase : la mobilisation et l'orchestration de la technologie répressive (23-30 juillet)}

24 Le mouvement étudiant mexicain est le résultat d'une réponse sociale aux représailles des autorités locales qui mettent en œuvre une sorte de "répression préventive ». Cette politique préventive était en vogue durant la période de la Guerre froide : elle devait garantir la tranquillité et la stabilité politiques, en réprimant les acteurs individuels et collectifs dérangeants pour le gouvernement autoritaire. "On réprime pour éviter le complot» indique Monsivaís (Varios Autores 2007: 32). À la tête du régime est fermement installé un président autocrate qui perçoit comme « bruissement menaçant ou intelligible » tout ce qu'il ne voit pas d'un bon œil (Varios Autores 2007 : 33).

Le gouvernement provoque et attribue la responsabilité des troubles aux groupes rebelles. Il a ainsi faussement accusé le Parti communiste mexicain (PCM) de transgression, au moment même où celui-ci réalisait des efforts désespérés de rapprochement avec le gouvernement en vue d'obtenir son inscription comme parti légal. En même temps, le gouvernement considère la révolte comme un moyen de marginaliser le PCM, au moins pendant la durée des $19^{\text {es }}$ Jeux Olympiques qui doivent débuter le 12 octobre à Mexico. Le discours de l'État se construit sur une simple argumentation : la révolte est un plan d'agitation et de subversion. Durant toutes les actions collectives postérieures, les autorités agissent selon les même intentions constantes: provoquer la division interne entre les réseaux et alliances obtenues, provoquer la violence pour justifier l'intervention de l'État, multiplier les infiltrations des mouvements pour identifier les leaders et les déstabiliser, provoquer une réaction des citoyens basée sur une fausse interprétation de la confrontation, contrôler les médias, les corps de police et l'armée. Les autorités étatiques utilisent l'émotion comme moyen pour contrôler la situation et mettre fin aux sympathies croissantes envers le mouvement : la peur, d'abord des étudiant-e-s et ensuite de la population de la ville.

Le mouvement se développe rapidement comme résultante de la répression provoquée par les autorités. Dans un premier temps, la répression ne réduit pas la participation; elle amplifie au contraire l'engagement et les attentes. Les réponses étudiantes augmentent à mesure que s'intensifie la répression des forces du maintien de l'ordre. Durant quatre jours (du 26 au 29 juillet), on assiste à l'occupation de lycées par l'armée, à des encerclements policiers, à des incendies de bus transformés en barricades, à des blessures contre des manifestants, à des arrestations d'activistes dont certains sont portés disparus ou meurent en prison. La violence de l'État est présente du début à la fin $\mathrm{du}$ mouvement. Le gouvernement cherche clairement la confrontation (Anguiano $2010: 75$ ) pour justifier l'encerclement et mater la révolte.

Comme nous l'avons mentionné, le rôle de la répression fonctionne comme un mécanisme de mobilisation-démobilisation. Les mesures répressives sont un acte de réprobation exercé par l'autorité sur les subalternes. En termes politiques, cette 
contrainte va de pair avec la pratique du consensus dans la construction de l'hégémonie (Gramsci in Portelli 1992). En fonction de son degré d'utilisation, il s'agit d'un acte d'assujettissement ayant pour objectif de soumettre des individus ou des groupes subordonnés. Mais tous les actes de répression n'ont pas ces effets. Selon les circonstances politiques et culturelles d'une configuration particulière, la réponse collective des subalternes peut varier. Un fait de répression peut générer l'éclatement de l'indignation et de l'agitation sociale. Dans le mouvement étudiant, on observe ce double mouvement, avec au départ une perception de la répression comme moteur de la mobilisation, à l'instar de ce qu'indique l'une des intégrantes du mouvement :

«Je pense que la force et l'importance du mouvement étudiant lui ont été données par la répression. Plus que n'importe quel discours politique, le fait même de la répression a politisé les personnes et a permis que la grande majorité participe activement aux assemblées $»^{4}$.

La caractéristique singulière du pouvoir telle qu'elle se donne à voir à partir des premiers affrontements avec les étudiant-e-s est un autre élément expliquant le processus de mobilisation-démobilisation. L'intolérance par le pouvoir de toute dissidence dans un contexte de transformations sociales et culturelles a un impact très clair sur la société mexicaine. Dans ce contexte, aucun fonctionnaire de l'État n'avait prévu la mobilisation étudiante. Lorsque le recteur de l'UNAM, Barros Sierra, met le drapeau du Mexique en berne le 30 juillet et appelle à la défense de l'autonomie universitaire à la suite de la destruction de la porte du lycée $\mathrm{n}^{\circ} 1$ de l'UNAM à coups de bazooka par l'armée, personne ne saisit clairement ce qu'il est en train de se passer (Rodríguez Kuri 2003 : 221 ), dans la mesure où il semblait aller de soi que tous les niveaux d'autorité étaient détenus par les élites, les élites universitaires incluses. Le gouvernement ne parvient pas non plus à comprendre la spirale de violence qu'il avait lui-même produite.

L'action de la police contre les lycéens (d'un lycée privé et d'un lycée de l'IPN) qu'elle avait affrontés lors d'une rixe génère l'indignation de nombreux secteurs. L'absence de compréhension de la situation politique par la police est à l'origine de l'extension de son attitude répressive à d'autres établissements d'enseignement supérieur et au PCM, de même qu'à l'UNAM et à l'IPN. À la répression répond la violence des étudiant-e-s. Une violence verticale (Bulhan 1985) se déchaine entre lycéen-ne-s et forces de l'ordre, qui provoque la présence de l'armée durant cinq jours dans le quartier universitaire du centre historique de la ville. L'armée force l'accès à un site de l'UNAM qui n'avait rien à voir avec le conflit initial. L'attitude du recteur Barros Sierra qui appelle au droit de manifester contre « le tir de bazooka » et le pouvoir présidentiel « sans se soumettre au règlement de la police qui imposait le dépôt d'une demande préalable de permis et une autorisation accordée par le gouvernement local» (Anguiano 2010: 89) donne aux étudiant-e-s une plus grande confiance en eux et une conscience du mérite de leur action protestataire. Tous ces acteurs se sentent soudainement autorisés à donner une réponse née de l'indignation et à s'unir au sein d'un même réseau contre une attitude d'autorité.

En effet, à partir de ce moment s'élaborent rapidement de fortes alliances entre des groupes qui s'étaient auparavant souvent opposés lors de rixes impliquant supporters et fraternités étudiantes à l'occasion de compétitions sportives (il existait en particulier une opposition très forte entre l'IPN et l'UNAM). Face à la répression de l'État, les étudiant-e-s de l'IPN, de l'UNAM, des écoles normales (Superior, para Profesores, Rurales), l'École nationale d'agriculture Chapingo, les universités privées 
(Iberoamericana, Anáhuac, El Colegio de México), les universités fédérales (Escuela Nacional de Antropología e Historia - ENAH) tissent des liens. En outre, les réseaux d'action intègrent plusieurs degrés d'enseignement (lycées, écoles préparatoires, facultés et écoles professionnelles), des enseignants, intellectuels et artistes, mais aussi des groupes urbains de colonias (quartiers) populaires, des bandes de jeunes des quartiers, des parents d'élèves. La participation des femmes est très importante (Cohen et Frazier 2004). Plus encore, des liens s'établissent entre groupes politiques illégaux et étudiant-e-s. La politique de l'État vise alors à rompre ce réseau d'alliances stratégiques, en premier lieu à travers la répression extensive et prolongée, mais aussi avec l'infiltration, les tentatives de cooptation de leaders et le contrôle des médias. Il optera par la suite pour la répression intensive.

\section{$2^{\mathrm{e}}$ phase : développement et intégration de répertoires de mobilisation et stratégies étatiques de fragmentation ( $1^{\text {er }}-28$ août)}

31 L'analyse des mouvements subversifs, dans la perspective du maintien de l'ordre, consiste à construire des formes contrastées de désarticulation de l'activité protestataire. La correspondance entre insurrection et mouvements sociaux est considérée comme ontologique pour l'intelligence militaire. Le travail du commodore D’Odorico consacré à « la guerre non conventionnelle » est en ce sens utile pour penser les éléments qui doivent être pris en compte par les forces armées, en particulier la première loi relative à la «connaissance des ennemis». Les formes de mobilisation, la planification et l'élaboration de stratégies sont liées à la compréhension des leaderships, la diversité des idéologies libertaires, la cooptation, les liens des groupes insurgés avec la société ou les communautés, l'instruction et l'éducation politiques, entre autres. Sur cette base, les forces armées doivent être capables « d'utiliser toutes les ressources disponibles pour décourager le plan insurrectionnel » (D'Odorico 2011b : 63 ; 2011a). C'est dans ce contexte que l'on peut expliquer la production de technologies répressives. Nous établirons ici la correspondance entre mobilisation et démobilisation à partir d'une analyse ayant quatre dimensions.

\section{Structure organisationnelle et leadership}

32 Le répertoire de mobilisation rassemble une combinaison de formes d'organisation et d'action politique. Une première initiative consiste à créer une structure dynamique, massive, de représentation démocratique de la part des étudiant-e-s. Le CNH est conçu comme lieu de centralisation et de définition de la politique pour l'ensemble du mouvement. Il est formé par près de 300 délégué-e-s de tous les établissements en grève. Chacune d'entre elles est, à son tour, un noyau de mobilisation, d'organisation et de débat au travers d'assemblées propres à chaque établissement où sont discutées la situation du conflit, la trajectoire du mouvement et où sont désigné-e-s les délégué-e-s au CNH. Là s'organisent les comités de lutte, conçus comme la partie exécutive du mouvement, en commissions presse et propagande, finances, cuisine et brigades. Certains leaders coïncident dans la caractérisation des acteurs du mouvement en signalant le $\mathrm{CNH}$ comme le "cerveau», les assemblées comme "le système circulatoire», les manifestations massives publiques comme «le muscle» et les 
brigades comme la courroie de transmission et les "points névralgiques » de création d'énergie et de construction de vastes réseaux sociaux ${ }^{5}$. Tant que fonctionne cette structure structurante, le mouvement continue comme entité vivante qui accroît ses horizons de participation. Pour les autorités, la définition du mouvement part de sa tête, c'est-à-dire des délégué-e-s qui composent le $\mathrm{CNH}$, en particulier de celles et ceux qui apparaissent publiquement au fur et à mesure du conflit. Pour le gouvernement, décapiter le mouvement équivaut à briser sa structure en vue de générer la démobilisation.

La démobilisation du mouvement est d'abord recherchée à travers la cooptation de leaders ou activistes ayant une certaine influence sur l'orientation de la lutte. La corruption étatique a pour objectif de mettre fin à l'identification entre les groupements et les leaders; l'ambition est alors de transformer le mouvement, en l'institutionnalisant sous les auspices du parti dominant. Le leadership du mouvement est collectif, représentatif, composé par des activistes ayant pour certains d'entre eux une expérience politique préalable. De fait, une majorité d'entre eux étaient préalablement membres d'organisations de gauche, du PCM, du courant spartakiste créé par l'écrivain José Revueltas suite à une scission avec le PCM, d'autres courants du trotskysme, du maoïsme et du guévarisme ou encore de partis institutionnels comme la droite Action Nationale et Démocrates chrétiens ${ }^{6}$.Tant que le mouvement se structure de manière horizontale, avec une direction politique ouverte à une représentation collective, les initiatives de démantèlement du gouvernement échouent. Quoi qu'il en soit, l'infiltration apparaît très délicate à gérer pour les mouvements.

\section{Médias et brigades : gagner des soutiens}

Une autre des réponses de l'État à l'essor du mouvement est le contrôle des médias. L'objectif est là de dissoudre le lien entre le mouvement étudiant et la population. La théorie des cadres (Hunt, Benford, et Snow 1994) montre comment émergent les champs identitaires (l'identification des attributs des groupes) pour les protagonistes, les opposants et les publics. Il s'avère que celui des publics est l'objectif le plus important des acteurs belligérants dans un espace de conflit. Aussi bien les protagonistes que les opposants cherchent à attirer les publics en direction de leurs constellations discursives. Les publics reçoivent l'information par différents moyens, mais les médias institutionnalisés généralement contrôlés par l'État jouent un rôle prépondérant. Tant que les médias sont contrôlés par l'État, c'est le discours et l'interprétation institutionnelle du mouvement qui est davantage relayé auprès de la population. Or, si la critique faite au mouvement est forte et partagée, le gouvernement peut recourir à des mesures politiques de répression et de dissuasion de la mobilisation, sans s'exposer au « jugement public » (Manin 1995).

Pour sa part, le mouvement cherche à contrecarrer l'interprétation officielle grâce à ses propres médias. Dans le cas du mouvement étudiant, en plus de la radio de l'UNAM, un élément fondamental qui permet de relayer le discours du mouvement auprès de la population urbaine de la ville de Mexico est la création de brigades politiques qui essaiment dans toute la ville. Elles sont généralement composées de 3,5 ou 10 étudiante-s très mobiles qui distribuent des tracts et des communiqués, réalisent des meetings éclair, demandent le soutien du public pour maintenir le mouvement, peignent les messages du mouvement sur les murs et les autobus ; les meetings éclairs se réalisent 
sur les marchés, dans des écoles, des fabriques, des bureaux, des carrefours, des parcs et des places; des chiens et des chats arborent des messages politiques. On peut voir l'importance des brigades dans le témoignage suivant :

« Tu sais, les brigades étaient la vie du mouvement. Les gens allaient aux manifestations grâce aux brigades. Pourquoi est-ce que tout le monde appuyait les étudiants? À cause des brigades, parce qu'avant on avait tracté dans les bus, les trolleybus, les marchés, les grands magasins, les ateliers, les coins de rue où l'on faisait des meetings éclairs et on filait comme des éclairs dès que l'on reniflait un policier. Ah! Comme je me souviens des brigades! $»^{7}$.

$\mathrm{du}$ pays pour obtenir des soutiens. Ceux-ci s'organisent à travers l'articulation à d'autres mobilisations, comme la Coalition des pères de famille, la Coalition des professeurs de l'éducation intermédiaire et supérieure Pro Libertés Démocratiques, l'Assemblée des écrivains et artistes et les actions collectives des étudiant-e-s et professeur-e-s universitaires de l'intérieur du pays ${ }^{12}$.

\section{Manifestations et rôle stratégique de l'espace public}

politiques du mouvement. Le gouvernement réagit en se lançant dans l'organisation de contre manifestations publiques censées démontrer la dimension minoritaire du mouvement en rébellion. Celles-ci visent également à générer des divisions parmi les étudiant-e-s et à semer la confusion parmi les participant-e-s. Conjointement aux actions de harcèlement que réalisent les membres de la FNET à l'IPN et à d'autres manifestations organisées sous les auspices du gouvernement par des groupements des jeunesses catholiques d'extrême-droite, les contre manifestations font clairement partie de la stratégie de démobilisation du gouvernement ${ }^{13}$.

Les manifestations sont des répertoires de mobilisation qui agissent comme rituels ou actes performatifs dirigés aussi bien à l'intérieur qu'à l'extérieur du mouvement. On voit l'impact politique qu'elles peuvent avoir en constatant l'ampleur des stratégies de discrédit que leur opposent les autorités par l'intermédiaire des médias officiels. Les 
actions répressives sont précisément orientées vers ce type de mobilisations. Elles s'ajoutent aux occupations policières des bureaux et des lieux de réunion des organisations dissidentes, comme par exemple le local du PCM, l'intervention de l'armée à l'UNAM et la violente occupation de l'IPN. La récupération des espaces physiques par l'intermédiaire de la violence est là encore une stratégie visant à la démobilisation. Les lieux de réunion, où se tiennent les assemblées générales, où s'organise l'activité des brigades, où se consolident les comités de lutte de chaque établissement d'enseignement supérieur participant au conflit, permettent une circulation de l'information et la prise de décisions collectives : il s'agit d'une " école de politisation, de réflexion collective et de socialisation" (Anguiano 2010: 83). Le gouvernement tente de contrôler ces lieux de formation politique afin de dissoudre les noyaux physiques du réseau de la mobilisation.

En plus de la première manifestation organisée par le recteur Barros Sierra le $1^{\mathrm{er}}$ août, le mouvement organise quatre manifestations et un rassemblement de masse sur la place de Tlatelolco (cf. tableau 3). La première manifestation est fondamentale dans la mesure où elle ouvre la voie à l'organisation des étudiant-e-s pour occuper les rues. Ensuite, la manifestation du 5 août montre un mouvement en voie de consolidation; les trois dernières manifestations sont profondément symboliques, en lien direct avec les revendications du mouvement. En effet, la manifestation du 13 août a pour objectif de clore la première marche sur le Zócalo (place de la Constitution) et de réaliser un meeting devant le Palais national, représentation symbolique du pouvoir. Comme mentionne l'un des participants, « le Zócalo était interdit à toute manifestation qui ne soutenait pas [le gouvernement] $\aleph^{14}$ et cette action d'effervescence symbolise un moment fort du processus de mobilisation. L'objectif est de montrer aux opposants que le mouvement est représentatif, de par son importance numérique, constitué par la majorité des étudiant-e-s; il s'agit aussi de donner à voir la légitimité de ses actions et de ses revendications. Cette manifestation rassemble plus de 250000 étudiant-e-s « craignant d'être brutalement réprimés, mais avec une ferme conviction... » (Martínez Della Rocca 2010 : 306).

\begin{tabular}{|l|l|}
\hline \multicolumn{2}{|l|}{ Tableau 3. Manifestations et participant-e-s au cours du mouvement étudiant de 1968 à Mexico } \\
\hline Manifestations & Nombre de participant-e-s \\
\hline $1^{\text {er }}$ août & 80000 \\
\hline 5 août & 100000 \\
\hline 13 août & 150000 \\
\hline 27 août & 250000 \\
\hline 13 septembre & 200000 \\
\hline
\end{tabular}

Note : Martínez Della Rocca (2010) estime que la participation aux manifestations du 13 août est de 250 000, à celles du 27 août de 500000 et à celles du 13 septembre de 300000 personnes.

Source : Anguiano 2010 


\section{Négociation politique et (dé)mobilisation}

La négociation politique, définie comme le processus par lequel se génère un accord ou la compréhension d'un problème public, peut être utilisée comme stratégie aussi bien de mobilisation ou de démobilisation, selon l'usage des formes de la négociation, de ses résultats et de la volonté politique des parties présentes. Si, institutionnellement, elle est définie comme une nécessité pour les gouvernements démocratiques de parvenir à des accords, ceci dépend en réalité des rapports de force des acteurs impliqués dans le conflit.

leur côté, les pouvoirs publics peuvent voir dans la négociation un moyen de déstructurer symboliquement les liens entre dirigeant-e-s et participant-e-s. À travers la négociation, il est possible de corrompre des leaderships, de coopter des dirigeant-e$\mathrm{s}$ ou d'établir une argumentation qui fasse prévaloir les intérêts individuels au détriment des intérêts collectifs. La négociation peut être utilisée pour ne pas négocier ou, en d'autres termes, pour exprimer publiquement la volonté de dialogue sans pour autant y souscrire, afin de ne pas reconnaître la légitimité des revendications de l'interlocuteur. Éviter la négociation consiste à nier la reconnaissance des revendications de l'autre. Cela peut par ailleurs provoquer la confusion au sein du mouvement voire provoquer la sécession de soutiens. Refuser la négociation avec l'acteur central du mouvement peut s'accompagner d'une stratégie de recherche d'allié chez une des parties du conflit afin de peser sur l'orientation du mouvement à travers une fausse négociation qui contribue à créer la confusion chez les membres du mouvement. Ainsi, la négociation peut être un élément clé de la démobilisation.

usqu' au 13 août (jour de la seconde manifestation, dont l'objectif est de souligner la représentation étudiante du mouvement), le président de la République reste silencieux face au CNH. Toutefois, selon le discours officiel, le gouvernement est en pourparlers avec la FNET, alignée sur la politique gouvernementale et le conflit est proche de sa résolution (Martínez Della Rocca 2010: 306). Le CNH se dit être prêt à dialoguer, mais en public.

La négociation peut être un mécanisme de division à l'intérieur d'un mouvement par définition pluriel. La théorie des cadrages montre comment sont élaborés les discours de diagnostic qui définissent le mouvement à l'interne, à partir des injustices perçues, du mérite des actions collectives et de l'identification des adversaires. Toutefois, elle laisse de côté le fait que ce diagnostic très général est le résultat de visions distinctes et de constellations discursives de groupes aux expériences politiques diverses. L'émergence d'un mouvement montre le succès des liens qui peuvent s'établir entre ces discours ; il montre également que ce succès est le résultat d'une lutte interne pour son hégémonie politique. Il n'en reste pas moins que le discours de diagnostic peut avoir des bases fragiles et que les opposants internes peuvent tenter de les miner via la négociation.

Le 23 août, les autorités soumettent une proposition peu claire de dialogue aux étudiant-e-s; elles le font indirectement, à travers la Coalition de professeurs. Cette situation provoque une série de divisions entre les groupes participant à la mobilisation. En effet, la proposition du mouvement d'un dialogue public génère plusieurs lignes de fracture en son sein. Les débats consacrés par exemple à « l'opportunité des manifestations unitaires au centre de la ville ou leurs déploiements par zones industrielles » (Anguiano 2010 : 94) s'accroissent. Se multiplient également 
les questions quant à la possible instrumentalisation du dialogue public ou quant aux conséquences des relations de contact direct que celui-ci implique avec les représentants du gouvernement. Le $\mathrm{CNH}$ « ne [peut] se décider à temps pour forcer une sortie négociée du conflit » (Anguiano 2010 : 94). De manière générale, se définissent deux positions principales : une représentée par un groupe plus modéré, lié au PCM, qui insiste sur la nécessité d'établir une négociation avec l'État; l'autre, portée par des groupes plus radicaux, certains d'entre eux contrôlant la coordination des brigades de la Cité universitaire, refuse la négociation ${ }^{15}$. En outre, les discussions en assemblée et la prise de décisions sont compliquées par des oppositions idéologiques liées aux conflits de la Guerre froide qui n'ont rien à voir avec le conflit étudiant: maoïstes et prosoviétiques se heurtent sur l'interprétation de la guerre sinosoviétique; de longs discours sont prononcés, avec des références ouvertes à Mao Tse Toung, Trotski, Lénine, Bakounine... ${ }^{16}$ Enfin, il existe des dissensions à l'intérieur du mouvement quant aux priorités et stratégies :

"Après avoir passé toute la journée dans une brigade, ça me faisait rire et me mettait en colère de revenir à la Cité universitaire et d'assister à une assemblée pour écouter les piques entre groupes et voir que les gars prenaient cinq heures pour aboutir à une satanée résolution. [...] Les théoriciens n'allaient pas dans les brigades; ils restaient enfermés en jacassant, en perdant leur temps. Ils parlaient, par exemple, des prisonniers politiques. Nous, les membres des brigades, on a commencé à comprendre ce qu'étaient les prisonniers politiques quand on a commencé à nous poursuivre sans que l'on ne fasse rien d'illégal... Au niveau de la base, on était tous amis : IPN, UNAM, Chapingo. Mais au $\mathrm{CNH}$, qui les maoïstes, qui les trotskos, qui les "pécés" [PC], quel bordel! J'étais de la base et je sais que l'on aurait accepté le dialogue avec qui que ce soit, mais au $\mathrm{CNH}$ non, on ne pouvait accepter le dialogue avec aucun fonctionnaire qui était dans la répression. Si le gouvernement entier était la répression! Dans les assemblées ils se lançaient dans des péroraisons de plus d'une heure sur Althusser, Marx et Lénine, mais ils ne disaient rien de ce [qui nous intéressait]. Que va-t-on faire demain? De vrais enculages de mouche! Ils avaient perdu par principe, parce que c'étaient des théoriciens qui passaient leur temps à nous expliquer pourquoi le gouvernement ne pouvait résoudre nos revendications $»^{17}$.

À côté des luttes pour l'obtention de soutiens, pour occuper l'espace public et - à travers la négociation - pour déstabiliser à l'interne le mouvement, l'État intervient encore par la diversification du recours à la violence : actions répressives ouvertement violentes, invasions dans les établissements d'enseignement supérieur, attaques par les membres des porras (groupes de choc promus par les autorités scolaires et gouvernementales) des barricades montées par les étudiant-e-s, dépôt de pierres dans les poubelles jouxtant le trajet des manifestations pour accuser ensuite les participante-s de violences durant les événements (pierres parfois récupérées des égouts par les activistes les plus radicaux $)^{18}$. Ces dispositifs répressifs se combinent avec d'autres actions massives, comme l'expulsion des étudiant-e-s de la place du Zócalo le 28 août, les occupations des installations universitaires et le massacre du 2 octobre à Tlatelolco. On assiste ainsi à la fin de cette deuxième phase à la mise en œuvre d'une répression institutionnalisée (González 2013). 


\section{$3^{e}$ phase : le début de l'éclatement ( 29 août - 2 octobre)} répressives, comme par exemple lors de l'expulsion des étudiant-e-s de la place du Zócalo par l'armée au prétexte d'un outrage au symbole de la patrie et aux traditions catholiques du peuple mexicain en pénétrant dans la cathédrale. Pour certains, ce moment marque les débuts d'une seconde étape caractérisée par l'escalade de la répression du mouvement : « l'État [repasse] à l'offensive » (Martínez Della Rocca 2010). Vient ensuite l'intervention de l'armée à l'UNAM et à l'IPN le 18 septembre et finalement l'assaut du 2 octobre à Tlatelolco. Toutefois, ces événements, même s'ils sont les plus marquants, ne sont pas les seuls cas de répression officielle; s'y ajoutent les attentats de groupes de porras et de paramilitaires dans les établissements en grève, de même que l'emprisonnement et les jugements partiaux de participant-e-s et de leaders étudiants, les interrogatoires sous torture et le harcèlement constant des prisonniers politiques par des prisonniers du droit commun sous le regard bienveillant des autorités pénitentiaires. La répression est la politique la plus tenace et au final efficace de la démobilisation.

À partir du 29 août, le gouvernement durcit sa stratégie. Des centaines de membres des brigades sont traqués et faits prisonniers. Un témoignage mentionne un étudiant arrêté dans la rue, puis abattu d'une balle dans la tête. Le policier auteur de cet acte est libéré deux mois plus tard. Des groupes paramilitaires mitraillent les établissements en grève (Martínez Della Rocca 2010 : 318). La ville est mise en état de siège, avec des centaines de rondes policières et des tanks patrouillant dans les rues.

L'image associée à l'étudiant-e est celle du-de la délinquant-e. La peur s'empare des étudiant-e-s mais aussi de leurs familles et des habitant-e-s de la ville ; les coûts de la mobilisation contribuent à la démobilisation.

\section{Escalade de la répression}

51 Sur la base de témoignes, nous considérons que la mobilisation étudiante atteint son apogée lors de la manifestation massive du 27 août. Nous situons le point d'inflexion du mouvement, qui marque une démobilisation persistante, au moment de l'expulsion des étudiant-e-s de la place du Zócalo le 28 août. Le mouvement commence alors à se fragmenter, au début presque imperceptiblement, sur la base d'un incident le 29 août, lors d'un meeting organisé par les autorités du District fédéral pour réaffirmer les symboles de la patrie et les valeurs religieuses traditionnelles «outragées " par les activistes. Lors de la manifestation, les agents du secteur public obligés d'assister à l'événement réagissent contre les autorités et transforment le meeting officiel en action protestataire des travailleurs de la fonction publique. L'acte est réprimé par des soldats et des tanks de l'armée mexicaine. La perception des étudiant-e-s est alors que la lutte pour le soutien de la population est gagnée et que la rupture du contrôle corporatif par les syndicats de la fonction publique est un symbole du changement. Toutefois, cet incident «influença certainement la décision gouvernementale de pousser vers une sortie militaire» (Anguiano 2010 : 91). Dès lors, le rapport de force se modifie.

European Journal of Turkish Studies, 17 | 2013 

proposition implique des risques tant pour le gouvernement que pour le mouvement. Les étudiant-e-s avaient invité des représentants de la chambre des députés à dialoguer le 20 août, ce que ces derniers refusèrent. Le gouvernement propose ensuite à deux reprises des pourparlers avec le $\mathrm{CNH}$, en cherchant, comme nous l'avons indiqué, à placer des représentants de la FNET comme interlocuteurs privilégiés et à passer outre la représentation véritable du mouvement ${ }^{22}$. Il propose alors, à travers le ministère de l'Intérieur, une première communication téléphonique pour établir le contact. Cette situation génère un fort débat à l'intérieur du CNH dans la mesure où cette manière de faire est vue comme un risque de déboucher sur des accords frauduleux, privés du contrôle de la base étudiante.

57 Pour le gouvernement, accepter le dialogue public demandé par les étudiant-e-s signifie rompre avec les pratiques politiques de manipulation, de clientélisme et de corruption construites depuis des décennies par les gouvernements postrévolutionnaires, à 
dominante autoritaire. Mais céder au dialogue et, plus encore, accepter les revendications du mouvement présupposerait d'encourager le surgissement d'autres mouvements. Il s'agirait d'un exemple évident de légitimation de la subversion à laquelle s'opposent les positions les plus conservatrices du gouvernement et des secteurs corporatifs placés sous la tutelle de l'État, en particulier le principal leader syndical du pays, ainsi que les groupes d'entrepreneurs nationaux et l'Église. On peut en ce sens comprendre le vif rejet par le ministère de l'Intérieur du dialogue public.

Face à l'impossibilité du dialogue, l'alternative de l'usage de la force publique, de l'encerclement massif et du contrôle militaire des sites d'enseignement supérieur semble être une option de plus en plus viable pour le gouvernement. Chaque mesure à considérer par les autorités est conditionnée par la temporalité, tant par le calendrier des fêtes de la patrie célébrées au mois de septembre que par le début imminent des Olympiades le 12 octobre.

Dans le contexte du dialogue, le dilemme se présente ainsi pour le mouvement: reprendre les cours sous la pression du recteur, ou bien continuer la grève jusqu'à satisfaction des revendications. La première option implique une démobilisation immédiate: l'activité des brigades ne pourrait plus être assurée et les ressources matérielles et en temps seraient par conséquent réduites, alors que se consolideraient le poids des cadres du mouvement et de la direction collective du CNH (Martínez Della Rocca 2010 : 334). Reprendre les cours suppose en outre la satisfaction d'au moins une partie des revendications pour éviter une perte trop importante de prestige du $\mathrm{CNH}$ et le déclin de la force sociale. L'autre option, pour laquelle opte l'assemblée, est de poursuivre l'action collective, avec pour conséquence d'augmenter les probabilités de répression, voire de décapitation du mouvement, de même que l'isolement total du $\mathrm{CNH}$ au sein de la communauté universitaire.

$\mathrm{Au}$ moment où le dialogue génère l'incertitude et la méfiance au sein du mouvement, le gouvernement agit sur deux plans. D'un côté, il construit un discours de dénigrement systématique du mouvement en l'accusant d'actes de délinquance orchestrés par une force invisible subversive provenant de l'extérieur du pays, une conjuration communiste et contre la nation ${ }^{23}$. Pour ce faire, il utilise les médias et certaines organisations de la structure corporative. De l'autre, tout en prônant officiellement la négociation, il utilise les forces du maintien de l'ordre pour réprimer brutalement le mouvement ${ }^{24}$.

61 Le gouvernement élabore alors un double objectif: d'un côté chercher la démobilisation à l'intérieur du mouvement, de l'autre, le décapiter en mettant ses leaders en prison. L'importance des leaderships dans les conflits sociaux est de premier ordre pour les autorités. Les mouvements cherchent fréquemment à réduire le risque de ciblage du leadership par les pouvoirs publics en minorant le caractère public des leaders. $\mathrm{Au}$ sein du mouvement étudiant, on cherche ainsi à construire des formes d'organisation plus horizontales et collectives, mais sans pour autant empêcher l'émergence de leaders au cours du processus de mobilisation, qui feront ensuite l'objet d'une répression minutieuse du gouvernement lors de la quatrième phase de la lutte.

\section{4 e phase : I'hécatombe ( 3 octobre -6 décembre)}

62 Comme nous l'avons mentionné, la démobilisation débute le 28 août. Peu perceptible au début, la peur s'installe ensuite de manière plus tenace. La répression de la 
manifestation du 13 septembre débouche sur l'occupation militaire de certains sites d'enseignement supérieur et sur l'arrestation de centaines de personnes. Mais la répression du 2 octobre va plus loin encore : des dizaines ( 350 selon le New York Times ${ }^{25}$ ) d'étudiant-e-s meurent sur le front de la lutte et un grand nombre d'activistes sont arrêtés et mis en détention, 200 parmi eux pendant plus de deux ans et demi, ce qui finit par décapiter la direction du mouvement. La peur prédomine; suscitée par les autorités. Un activiste signale qu'après le massacre la désorganisation est quasi totale :

« [Les étudiants] cherchaient à se réunir de nouveau et agir, parfaitement terrifiés, battus, au jour le jour, dans des conditions très difficiles parce qu'ils devaient se réunir dans les rues, les parcs, les écoles, là où ils pouvaient, et ils ont commencé à se désagréger... le contact avec les bases se perdait... il était très difficile de faire des réunions $»^{26}$.

En découle une démobilisation accélérée. L'esprit festif et d'assurance de la victoire se transforme radicalement. Un activiste relate par exemple que le lendemain, la réunion organisée à la Cité universitaire rassemble 17 personnes $^{27}$. Pour une autre adhérente au mouvement, toute manifestation publique se révèle impossible avec un tel niveau de répression : «Il n'y avait rien d'autre à faire pour sortir de cette situation. Où et avec qui pouvions-nous faire des manifestations massives après le 2 octobre? La ville était terrifiée ${ }^{28}$. Par ailleurs, le mouvement n'arrive plus à envisager une satisfaction, ne serait-ce que très partielle, de ses revendications.

La répression ne renvoie pas seulement à la dispersion des concentrations publiques, mais également aux mécanismes d'interrogation et de torture des détenu-e-s. Plus de 2000 personnes sont arrêtées et mises en détention le 2 octobre, s'ajoutant aux centaines de détenu-e-s depuis le 27 août et à la suite de l'occupation de l'UNAM. Aux tortures, aux simulations d'exécution et de castration s'ajoutent d'autres intimidations, enlèvements et violences contre les familles des détenu-e-s (Anguiano 2010) :

«J'ai passé [ma détention] en travaillant et en pissant du sang à cause des coups. Ils m'ont mis dans une cellule d'un mètre et demi sur deux mètres pendant un mois. Ils me donnaient une tasse de [boisson à base de maïs] le matin et une autre l'aprèsmidi. Sans couverture, ils m'ont mis un seau pour que je fasse mes besoins et ne le changeaient jamais. Tu sais ce que c'est? ... J'entendais : "Il est dix heures du soir !" et moi, imagine, comme si j'étais un chien de Pavlov. Ils viennent me chercher, ils vont me péter la gueule. Alors je me faisais tout petit, je commençais à trembler et à pleurer, pleurer $»^{29}$.

Ce témoignage, comme bien d'autres, montre l'impact de la prison, mais aussi la construction d'une mémoire du travail militant, des leaders martyrs, où disparaissent les actions des femmes activistes. Cohen et Frazier (2004: 620-621) indiquent clairement en ce sens que « $[t]$ out comme les militants emprisonnés de 68 dépendaient dans une large mesure de l'effort ignoré des visiteuses pour leur soutien physique et émotionnel, les quêtes idéalistes des étudiants sont subventionnées par le travail nécessaire mais invisible d'autres. [...]. En définitive, la possibilité qu'un étudiant mâle "étudie, lutte et travaille" nécessite la contribution reproductive spécifique de la femme, contribution que son manque de notoriété rend inappréciable (et facilement oubliable). Les restrictions de classe et de genre placent ces nobles activités hors d'atteinte de la majorité du peuple. Ces valeurs, dès lors, reflètent mieux ce que les leaders mâles ont généré et partagé dans l'espace collectif de la prison, l'utopie intellectuelle homosociale étant incarnée dans leurs narrations, où la souffrance corporelle créait le contexte pour le développement transcendant et le sens de la communauté ». Ce travail sur les émotions comme moteur d'une identification 
commune est ainsi stratégiquement mobilisé également par les activistes en faveur d'une poursuite de la mobilisation.

Les objectifs et stratégies du mouvement se transforment radicalement. Au sein de la direction du mouvement, le profit sociopolitique des dirigeant-e-s se modifie également, dans la mesure où la majorité des leaders sont en prison. Le Parti communiste et la Jeunesse communiste acquièrent une place prépondérante à la tête du mouvement. La seule possibilité que la nouvelle direction puisse envisager est de générer les conditions internes pour mettre fin à la grève étudiante.

67 Le mouvement réduit l'amplitude des revendications à la liberté des prisonniers politiques et au retrait de l'armée de l'IPN comme condition au dialogue. Ce qui subsiste du mouvement pousse à la démobilisation et ainsi à son isolement une fois de plus. Demeure une structure déstructurée d'assemblées avec une participation minime d'activistes, laissant sur la touche de nombreux-ses étudiant-e-s qui cessent de participer par peur et par découragement. Les témoignages des protagonistes coïncident quant à la sensation de défaite, de frustration, d'amertume et de ressentiment qui s'empare du mouvement. Ce dernier cesse d'être perçu comme un moyen d'atteindre un meilleur avenir. Les autorités parviennent ainsi à rompre le lien entre le cercle des participant-e-s les plus engagé-e-s et actif-ve-s et ceux-lles moins expérimenté-e-s mais majoritaires du mouvement.

Les autorités modifient dès lors leur stratégie. Les Jeux olympiques se déroulent sans problème et 34 jours après l'occupation des sites de l'IPN, l'armée se retire, à l'exception de la Vocacional 7 qui perd son statut de centre d'enseignement supérieur. La pression se maintient via des porte-paroles, comme le président du PRI, qui diffuse des menaces voilées contre les établissements d'enseignement supérieur.

69 À partir du 19 novembre, malgré la volonté de certains groupes et activistes, les assemblées commencent à céder face à la pression exercée sur le mouvement pour mettre fin à la grève. La décision n'est pas facile à prendre. Les groupes les plus radicaux mettent l'accent sur les émotions pour trouver un appui dans l'assistance clairsemée. Les morts et prisonniers politiques sont élevés au rang de martyres dans un cadre affectuel afin de maintenir la grève jusqu'au bout et de tenter d'élargir à nouveau le mouvement dans l'attente d'une action spontanée qui pourrait le redynamiser; la lutte adopte alors le slogan: "En mémoire de celles et ceux qui ont perdu la vie pour défendre la liberté ». Les assemblées qui auparavant, malgré la durée des réunions et les tensions en leur sein, constituaient le système nerveux du mouvement, s'opposent de plus en plus fréquemment aux comités de lutte et aux activistes de gauche, ces derniers finissant par les dominer totalement.

70 Les répertoires de la mobilisation qui fonctionnaient précédemment comme facteurs d'agrégation et d'intégration se transforment en éléments de démobilisation : la grève, produit de l'effervescence de milliers de personnes, devient une charge pour ceux qui veulent la poursuivre car elle ne permet plus d'attirer de nouveaux activistes et sert d'argument à la répression. En outre, les brigades ne peuvent plus agir ; il devient ainsi impossible d'obtenir des ressources et du soutien. Cette situation conduit la nouvelle direction du $\mathrm{CNH}$ à repenser les formes de la lutte du mouvement, en particulier concernant la poursuite de la grève.

71 Jusqu'à la fin, les divisions entre groupes accentuent la décomposition du mouvement. La décision finale du $\mathrm{CNH}$ de mettre fin à la grève le 21 novembre est considérée par 
certains représentants de la faculté de Philosophie comme une décision unilatérale ; ces derniers proposent de ne pas reconnaître l'autorité du $\mathrm{CNH}$. Au contraire, ceux qui soutiennent la décision la justifie par l'importante désertion des étudiant-e-s.

La combinaison des stratégies des autorités parvient ainsi à mettre fin au mouvement. Le $\mathrm{CNH}$ est incapable de trouver une réponse à la violence officielle et la répression contribue à la démobilisation. Le mouvement éclate et perd sa cohésion interne. Divers groupes commencent à agir au nom du $\mathrm{CNH}$ provoquant une perte de prestige ou plus de confusion encore. Certains représentants du CNH ont des contacts avec des fonctionnaires du gouvernement sans en informer les autres membres, générant un processus de délégitimation de celui-ci. Les actions d'infiltration, de division et de cooptation des étudiant-e-s donnent peu à peu leurs fruits, contribuant à l'affaiblissement du mouvement. Le CNH se transforme ainsi en organisme "d'avantgarde " sans base sociale. Ce sont finalement les groupes politiques qui imposent la reprise des cours (Martínez Della Rocca 2010 : 439-440). Les ressources et les répertoires de la mobilisation se transforment, dans une sorte de cercle vicieux, en facteurs de démobilisation.

\section{Conclusion : les dimensions plurielles de la répression}

Le 68 mexicain souligne la nécessité d'une analyse contextualisée de la dynamique de la mobilisation-démobilisation et du rôle de la répression. D’un côté, la répression peut stimuler la participation, l'action du gouvernement contribuant à rassembler des personnes et des organisations face à un adversaire commun. De l'autre, cette même répression peut conduire à la démobilisation, surtout si à la répression visible s'ajoute une répression "diffuse ». Comme le mentionnent Combes et Fillieule (2011: 1057) : "De même que le pouvoir ne saurait se mesurer à son exercice effectif, la répression doit prendre en compte aussi la menace, l'incapacitation et le renseignement ». Le 68 mexicain montre clairement, en plus des actions visibles, l'importance de cette répression souterraine.

L'augmentation constante de la répression de l'État face aux étudiant-e-s révèle les limites d'un gouvernement peu préparé à lutter contre un mouvement qui sort des canons établis de la culture politique postrévolutionnaire. Finalement, ce qui contribue au dénouement violent est que l'État considère qu'il affronte « un ennemi autonome qu'il ne peut contrôler avec ses méthodes clientélistes " (Anguiano 2010: 97). Le déroulement du processus illustre comment, à l'aide des techniques et des stratégies du gouvernement, on passe du risque au haut risque. La perception, de la part des étudiant-e-s, des coûts de l'engagement dans la lutte se modifie à la fin du mois d'août, face à l'action gouvernementale incessante de répression visible, de minage des réseaux d'appui et des alliances du mouvement et de discrédit vis-à-vis des citoyens, ce qui contribue à sa fragmentation et à la démobilisation.

La lutte étudiante au Mexique révèle le rôle central des émotions dans ce double processus de mobilisation et démobilisation. À l'indignation initiale face à la répression provenant de l'armée et de la police se superpose, pour ensuite s'imposer, la peur: arrestations, infiltrations, intimidations physiques et verbales, violences conduisant parfois à la mort. Ces émotions sont ainsi liées aux perceptions des activistes s'agissant de la possibilité ou non de poursuivre la lutte. Mais le travail sur les émotions joue également à d'autres niveaux de la mobilisation. S'agissant de femmes activistes en 
particulier, le travail affectuel permet une désectorisation de la lutte et un accroissement de ses bases sociales (Cohen et Frazier 2004). Ce travail est également très important lors des derniers soubresauts du mouvement lorsque l'on en appelle aux morts et aux prisonniers politiques pour poursuivre la lutte.

En dépit de son dénouement tragique, le mouvement étudiant a eu au moins trois conséquences majeures. D'abord, il a montré à la face du monde les limites du système politique mexicain et de la légitimité d'un gouvernement de parti unique. Ensuite, il a ouvert le champ des possibles de la participation politique : "dans sa trajectoire brève et fulgurante le mouvement étudiant n'est pas seulement politique mais aussi, très largement, social et culturel, en rendant visible la possibilité de la citoyenneté et en construisant diverses alternatives, la première d'entre elles étant la certitude d'autonomie possible en marge des corporations et des contrôles du gouvernement » (Monsivaís 2005: 15). Il faudra certes encore des années pour que le pluralisme politique et la participation citoyenne s'affirment, notamment lors du mouvement étudiant de 1986. Cependant, le 68 mexicain constitue une brèche dans l'autoritarisme étatique et dans la perception de l'engagement citoyen, en particulier pour les femmes (Cohen et Frazier 2004). Finalement, la démobilisation ne signifie pas la disparition des réseaux d'activistes, réseaux «dormants » qui se réactivent à plusieurs reprises : lors d'autres luttes étudiantes, mais également dans la création de partis politiques et d'organisations en lutte pour la transition démocratique au Mexique.

Annexe : Chronologie du mouvement étudiant de 1968

\begin{tabular}{|c|c|}
\hline Date & Événement \\
\hline 22 juillet & $\begin{array}{l}\text { Répression policière contre des étudiant-e-s et enseignant-e-s des écoles } \\
\text { préparatoires, résultats d'une rixe entre jeunes assistant à un match de football. }\end{array}$ \\
\hline 26 juillet & $\begin{array}{l}\text { La manifestation qui commémore la Révolution cubaine conflue avec une } \\
\text { manifestation organisée par des étudiant-e-s qui protestent contre la police. } \\
\text { Toutes deux sont durement réprimées. }\end{array}$ \\
\hline 28 juillet & $\begin{array}{l}\text { Les combats entre les étudiant-e-s et la police continuent dans le quartier } \\
\text { universitaire du centre historique. La grève se généralise dans les établissements } \\
\text { d'enseignement supérieur. }\end{array}$ \\
\hline 30 juillet & $\begin{array}{l}\text { Javier Barros Sierra hisse le drapeau en berne en protestation de la violation de } \\
\text { l'autonomie universitaire. }\end{array}$ \\
\hline 1er août & A lieu la première grande marche convoquée par le recteur Javier Barros Sierra. \\
\hline 2 août & Le CNH est créé. \\
\hline 5 août & 100000 étudiant-e-s de l'IPN réalisent une manifestation massive. \\
\hline 13 août & 250000 étudiant-e-s et autres citoyen-ne-s assistent à la marche du Zócalo. \\
\hline 27 août & 300000 personnes à la manifestation du musée d'Anthropologie au Zócalo. \\
\hline 28 août & L'armée déloge les étudiant-e-s du Zócalo. \\
\hline
\end{tabular}




\begin{tabular}{|c|c|}
\hline 1er septembre & $\begin{array}{l}\text { Le président de la République, Gustavo Díaz Ordaz, donne son IVe rapport de } \\
\text { gouvernement et aborde le problème étudiant avec des menaces voilées } \\
\text { d'intervention militaire. }\end{array}$ \\
\hline 13 septembre & $\begin{array}{l}250000 \text { personnes assistent à la marche du Silence, la plus significative du } \\
\text { mouvement. }\end{array}$ \\
\hline 18 septembre & $\begin{array}{l}\text { La nuit, l'armée occupe la cité universitaire; des centaines d'étudiant-e-s sont } \\
\text { arrêté-e-s. }\end{array}$ \\
\hline 24 septembre & $\begin{array}{l}\text { L'armée occupe le centre d'enseignement de l'IPN après plusieurs heures de tirs } \\
\text { croisés avec les étudiant-e-s. }\end{array}$ \\
\hline 30 septembre & Les autorités rendent les installations de la cité universitaire. \\
\hline $\begin{array}{l}2 \text { octobre } \\
\text { (matin) }\end{array}$ & Début des discussions entre étudiant-e-s et représentant-e-s du gouvernement. \\
\hline $\begin{array}{l}2 \text { octobre } \\
\text { (après-midi) }\end{array}$ & $\begin{array}{l}\text { Un meeting se tient à la place de Tlatelolco. L'armée et le bataillon Olimpia le } \\
\text { répriment brutalement et tuent des dizaines d'étudiant-e-s. }\end{array}$ \\
\hline 12 octobre & $\begin{array}{l}\text { Inauguration des XIXes Jeux olympiques qui ont comme devise «Tout est } \\
\text { possible dans la paix ». }\end{array}$ \\
\hline 24 octobre & $\begin{array}{l}\text { Entre le } 26 \text { juillet et le } 24 \text { octobre ont lieu } 5000 \text { arrestations. } 10 \% \text { (500) des } \\
\text { personnes arrêtées sont incarcérées. }\end{array}$ \\
\hline 27 octobre & Fin des Jeux olympiques. \\
\hline 11 novembre & $\begin{array}{l}\text { Après plusieurs tentatives pour maintenir le mouvement, il est convenu de la } \\
\text { nécessité que le } \mathrm{CNH} \text { appelle à cesser la grève lors d'une réunion entre } \\
\text { représentant-e-s d'étudiant-e-s, d'enseignant-e-s et du recteur. }\end{array}$ \\
\hline 6 décembre & Le CNH est officiellement dissout. \\
\hline
\end{tabular}

\section{BIBLIOGRAPHIE}

Álvarez Garín, Raúl (1998), La estela de Tlatelolco : una reconstrucción histórica del movimiento de 1968, Mexico, Ítaca.

Anguiano, Arturo (2010), « México 68. Irrupción de la sociedad en la política », in López Gallego, Alejandro ; López-Saavedra, Nicolasa ; Tamayo, Sergio ; Torres, Ricardo (dir.), Yo no estuve ahí pero no olvido. La protesta en estudio, VII taller internacional de etnografía urbana y cultura política "Análisis de la Protesta", set-nov. 2008, México, Universidad autónoma metropolitana, Mexico, Universidad autónoma metropolitana, pp. 73-99. 
Bertaux, Daniel (2005 [1997]), Le Récit de vie, Paris, Armand Colin, coll. L'Enquête et ses méthodes 128.

Brockett, Charles D. (2005), Political Movements and Violence in Central America, New York, Cambridge University Press, coll. Cambridge studies in contentious politics.

Brockett, Charles D. (1993), « A Protest-Cycle Resolution of the Repression/Popular-Protest Paradox ", Social Science History 17 (3), pp. 457-484. URL: http://www.jstor.org/stable/1171433

Broqua, Christophe ; Fillieule, Olivier (2009), « Act Up ou les raisons de la colère », in Traïni, Christophe (dir.), Émotions... mobilisation!, Paris, Presses de Sciences Po, coll. Sciencespo, Sociétés en mouvement.

Bulhan, Hussein Abdilahi (1985) Frantz Fanon and the Psychology of Oppression, New York, Plenum Press.

Cohen, Deborah ; Frazier, Lessie Jo (2004), « México 68 : hacia una definición del espacio del movimiento. La masculinidad heroica en la cárcel y las "mujeres" en las calles ", Estudios Sociológicos 22 (3), pp. 591-623. URL: http://www.jstor.org/stable/40420845

Combes, Hélène ; Fillieule, Olivier (2011), « Repression and Protest. Structural Models and Strategic Interactions ", Revue française de science politique (English) 61, pp. 1-24. DOI: http:// dx.doi.org/10.3917/rfsp.616.1047

Cruz Flores, Karina (2011), « La participación de la mujer universitaria en el movimiento estudiantil de 1968 en México ", in Memoria del XI congreso nacional de investigación educativa, historia e historiografía de la educación, URL : www.comie.org.mx/congreso/memoriaelectronica/ v11/docs/area_09/0925.pdf, dernier accès : 29 janv. 2014.

Diani, Mario (2004), « Networks and Participation », in Snow, David A.; Soule, Sarah A.; Kriesi, Hanspeter (dir.), The Blackwell Companion to Social Movements, Malden, Blackwell Publishing, coll. Blackwell companions to sociology ; pp. 339-359. URI: http://dx.doi.org/10.1002/9780470999103.ch15 D’Odorico, José C. (2011a), « La guerra no convencional III : la guerrilla en la guerra prolongada », Air \& Space Power Journal en Español 23 (1), pp. 77-89, URL : http://www.airpower.au.af.mil/ apjinternational/apj-s/2011/2011-1/2011_1_08_dodoricoa_s.pdf, dernier accès : 29 janv. 2014.

D’Odorico, José C. (2011b), « La guerra no convencional V : la guerra civil difiere de la subversiva ", Air \& Space Power, Journal en Español 23 (3), pp. 59-72. URL: http:// www.airpower.au.af.mil/apjinternational/apj-s/2011/2011-3/2011_3_09_racine_s_a.pdf

Echevarría, Nicolás (2008), série documentaire « El Memorial del 68 », Mexico, UNAM, URL :

$1^{\text {re }}$ partie : http://youtu.be/AtVMmxclMsk ;

$2^{\mathrm{e}}$ partie : http://youtu.be/DmLQ3t0uoP8;

$3^{\text {e }}$ partie : http://youtu.be/7PDUxXFtEok ;

$4^{\mathrm{e}}$ partie : http://youtu.be/252Ls6s5ZvQ ;

$5^{\mathrm{e}}$ partie : http://youtu.be/jo5hjc8-1VM, dernier accès : 9 février 2014

Editorial Estudiantes (1970), Los procesos de México 68. Acusaciones y defensa, Mexico, Ed. Estudiantes.

Fernandez, Roberto M.; McAdam, Doug (1988), « Social Networks and Social Movements: Multiorganizational Fields and Recruitment to Mississippi Freedom Summer », Sociological Forum 3 (3), pp. 357-382. URI: http://dx.doi.org/10.1007/BF01116431

Fillieule, Olivier (2013), « Demobilization », in The Wiley-Blackwell Encyclopedia of Social and Political Movements, DOI: http://dx.doi.org/10.1002/9780470674871.wbespm064 , dernier accès: 29 janv. 2014. 
Fillieule, Olivier (2010), « Some Elements of an Interactionist Approach to Political Disengagement », Social Movement Studies 9, pp. 1-15. URI: http://dx.doi.org/ $10.1080 / 14742830903442436$

Fillieule, Olivier (2005), « Temps biographique, temps social et variabilité des rétributions », in id. (dir.), Le Désengagement militant, Paris, Belin, coll. Sociologiquement, pp. 17-47.

González Marín, Silvia (dir.) (2003), Diálogos sobre el 68, Textos de las reuniones de trabajo organizadas por el Instituto de investigaciones bibliográficas, 2-4 sept. 1998, Mexico, UNAM.

González Villarreal, Roberto (2013), Historia de la desaparición. Nacimiento de una tecnología represiva, Mexico, Editorial Terracota, coll. Debate.

Goodwin, Jeff; Jasper, James M.; Polletta, Francesca (2004), « Emotional Dimensions of Social Movements ", in Snow, David A.; Soule, Sarah A.; Kriesi, Hanspeter (dir.), The Blackwell Companion to Social Movements, Oxford, Blackwell, coll. Blackwell Companions to Sociology, pp. 413-431, DOI: http://dx.doi.org/10.1002/9780470999103.ch18, dernier accès: 29 janv. 2014.

Gottraux, Philippe (1997), « Socialisme ou barbarie » : un engagement politique et intellectuel dans la France de l'après-guerre, Lausanne, Payot, coll. Sciences politiques et sociales.

Hobsbawn, Eric (2000), Historia del siglo XX, Barcelone, Crítica.

Hunt, Scott A.; Benford, Robert D; Snow David A. (1994), « Identity Fields: Framing Processes and the Social Construction of Movement Identities », in Laraña, Enrique; Johnston, Hank, Gusfield, Joseph R. (dir.), New Social Movements: From Ideology to Identity, Philadelphia, Temple University Press, pp. 185-208.

Jasper, James M. (1998), « The Emotions of Protest: Affective and Reactive Emotions in and around Social Movements ", Sociological Forum 13 (3), pp. 397-424. URL: http://www.jstor.org/ stable/684696

Leclercq, Catherine ; Pagis, Julie (2011), « Introduction », dossier « Les incidences biographiques de l'engagement », Sociétés contemporaines 4 (84), pp. 5-23. URI: http://dx.doi.org/10.3917/soco. 084.0005

Manin, Bernard (1995), Principes du gouvernement représentatif, Paris, Calmann-Lévy, coll. Liberté de l'esprit.

Martínez Della Rocca, Salvador (2010), Centenario de la UNAM. Estado y universidad nacional. Cien años de conciliaciones y rupturas, Mexico, Universidad de Guadalajara/Secretaría de Educación de la Ciudad de México/Miguel Ángel Porrúa.

McAdam, Doug (1999) « The Biographical Impact of Activism », in Giugni, Marco; McAdam, Doug; Tilly, Charles (dir.), How Social Movements Matter: Theoretical and Comparative Studies on the Consequences of Social Movements, Minneapolis, University of Minnesota Press, coll. Social movements, protest, and contention 10, pp. 119-146.

McAdam, Doug (1986), « Recruitment to High-Risk Activism: The Case of Freedom Summer », The American Journal of Sociology 92 (1), pp. 64-90. URL: http://www.jstor.org/stable/2779717

McAdam, Doug (1982), Political Process and the Development of Black Insurgency (1930-1970), Chicago, The University of Chicago Press.

Monsivaís, Carlos (2005), “No sin nosotros”: los días del terremoto 1985-2005, Mexico, Era, coll. Bolsillo Era.

Poniatowska, Elena (1971), La noche de Tlatelolco. Testimonios de historia oral, Mexico, Era, coll. Biblioteca Era. 
Portelli, Hugues (1992 [1973]), Gramsci y el bloque histórico, Mexico, Siglo XXI.

Récappé, Bénédicte (2008), Raison, émotion, institution. Comprendre les mobilisations étudiantes face à des régimes autoritaires : Hongrie, 1956, Mexique, 1968, Thèse de doctorat en science politique, IEP de Bordeaux.

Rodríguez Kuri, Ariel (2003) « Los primeros días. Una explicación de los orígenes inmediatos del movimiento estudiantil de 1968 », Historia Mexicana 53 (1), pp. 179-228, URL : http:// www.jstor.org/stable/25139488, dernier accès : 30 janv. 2014.

Snow, David A.; McAdam, Doug (2000) «Identity Work Processes in the Context of Social Movements: Clarifying the Identity/Movement Nexus ", in Stryker, Sheldon; Owens, Timothy J.; White, Robert W. (dir.), Self, Identity, and Social Movements, Minneapolis, University of Minnesota Press, coll. Social movements, protest, and contention 13, pp. 41-67.

Sommier, Isabelle (2010) « Les états affectifs ou la dimension affectuelle des mouvements sociaux ", in Fillieule, Olivier ; Agrikoliansky, Eric ; Sommier, Isabelle (dir.), Penser les mouvements sociaux. Conflits et contestation dans les sociétés contemporaines, Paris, La Découverte, coll. Recherches, pp. 185-202.

Tamayo, Sergio (1998) « Modernización y ciudadanía. El estado, los empresarios y el PAN frente al movimiento estudiantil de 1968 ", Sociológica 13 (38), pp. 49-81, URL : www.redalyc.org/ articulo.oa?id=305026670004, dernier accès : 30 janv. 2014.

Tamayo, Sergio (1999), Los veinte octubres mexicanos. La transición a la modernización y la democracia, 1968-1988 : ciudadanías e identidades colectivas, Mexico, Editorial UAM, coll. de Estudios urbanos.

Traïni, Christophe ; Siméant, Johanna (2009) « Pourquoi et comment sensibiliser à la cause ? ", in Traïni, Christophe (dir.), Emotions... Mobilisation!, Paris, Presses de Sciences Po, coll. Sociétés en mouvement, pp. 11-34.

Varios Autores (2007), ¡No se olvida! Testimonios sobre el movimiento del 68. Antología, Mexico, Secretaría de Cultura del GDF.

Wiltfang, Gregory L.; McAdam, Doug (1991), « The Costs and Risks of Social Activism: A Study of Sanctuary Movement Activism », Social Forces 69 (4), pp. 987-1010, URL: http://www.jstor.org/ stable/2579299, dernier accès: 30 janv. 2014.

\section{NOTES}

1. Entretien de Marcelino Perelló Vals, in Echevarría 2008, $5^{\mathrm{e}}$ partie, $1: 18-1: 20$.

2. Nous avons consulté des entretiens effectuées par Tamayo $(1998 ; 1999)$ ainsi que par Echevarría (2008), réalisés entre 2006 et 2007 ( $n=57)$, disponibles au Centre culturel universitaire de Tlatelolco, ainsi que des témoignages repris dans des sources secondaires entre le début des années 1970 (Poniatowska 1971) et la seconde moitié des années 2000 (González Marín 2003 ; Varios Autores 2007). Toutefois, le recours à ces sources est limité, dans la mesure où se pose à la fois le problème de la sélection par les chercheuses et chercheurs des paroles retenues (comme nous le verrons dans la suite de cet article, les entreprises mémorielles sont principalement le fait d'hommes activistes occupant une position influente au sein du mouvement), du contexte de l'entretien et des reconstructions subjectives des enquêté-e-s. Par rapport à ce dernier élément, nous rejoignons Bertaux lorsqu'il indique, dans un autre contexte, que s'il existe des "médiations subjectives et culturelles entre l'expérience vécue "brute" et sa mise en récit ", on ne peut considérer pour autant que l'acteur ne fait pas état de son propre parcours et, surtout, que "[l]'intervention des médiations signalées ne touche guère à la structure diachronique des 
situations, événements et actions qui ont jalonné ce parcours. Pour employer une métaphore, son "dessin" est bien restitué ; en revanche la remémoration peut en modifier rétrospectivement les couleurs » (2005:40-41).

3. Une chronologie de la lutte étudiante est consultable en annexe.

4. Carolina Pérez Cicero, étudiante de la faculté de Philosophie et Lettres de l'UNAM, in Poniatowska $1971: 16$.

5. Cf. le témoignage de Marcelino Perelló in Echevarría 2008, $2^{\text {ème }}$ partie, 13 :00-14:00.

6. Témoignage de Miguel Yoldi Marín, in González Marín 2003 : 86.

7. Salvador Martínez Della Rocca, du comité de lutte de la faculté des Sciences de l'UNAM, in Poniatowska $1971: 30-31$.

8. Pour Arturo Anguiano, activiste du mouvement, les étudiante-e-s ont, avec les brigades, « récupéré l'espace public, revendiquant la ville dans la pratique » (Anguiano 2010 : 80).

9. Comme le soulignent clairement les auteures, les actions des femmes, moins présentes dans les comités, sont largement minorées dans la construction de la mémoire historique tandis que le travail militant réalisé par les hommes activistes est magnifié.

10. Avec l'argent obtenu les activistes achètent des équipements de sonorisation, des hautparleurs, des génératrices, des projecteurs, etc. (témoignage de Raúl Álvarez Garín, in Varios Autores $2007: 52$ ).

11. Témoignage de Raúl Álvarez Garín (Varios Autores 2007 : 58).

12. Les grèves n'éclatent pas uniquement dans les universités du centre du pays : 70 écoles de 19 États de la République sont concernées (Jardón, in Anguiano 2010 : 82).

13. Ces groupements adhèrent au Mouvement universitaire d'orientation rénovée (Movimiento Universitario de Renovada Orientación, MURO) fondé en 1961, de même qu'au Front universitaire anticommuniste (FUA) créé quelques années auparavant dans la ville de Puebla.

14. Témoignage de Luís González de Alba in Echevarría 2008, $2^{\text {ème }}$ partie, 12 :00-12:10

15. Témoignage de Raúl Álvarez Garín in Varios Autores $2007: 61$.

16. Témoignage de Miguel Yoldi Marín in González Marín 2003 : 106. Voir également celui de Paco Ignacio Taibo II in Varios Autores 2007.

17. Salvador Martínez Della Rocca, dit Pino, du comité de lutte de la faculté des Sciences de l'UNAM, in Poniatowska $1971: 35$.

18. Témoignage de Jaime García Reyes in Varios Autores $2007: 22$.

19. Témoignage de Marcelino Perelló in González Marín 2003 : 70-71.

20. Témoignage d'Arturo Martínez Nateras in González Marín $2003: 142$.

21. Echevarría $2008,2^{\text {ème }}$ partie, 30 :00-31:28.

22. Témoignage de Raúl Álvarez Garín in Varios Autores 2007 : 63.

23. Les accusations à l'encontre des universitaires sont également directes, touchant aussi le recteur Barros Sierra. Sous la pression, le recteur donne sa démission à la junte de gouvernement de l'UNAM. Celle-ci est refusée, notamment grâce aux nombreux témoignages de sympathie d'instances universitaires et de personnalités publiques. Le retrait de l'armée de l'université découle en partie des pressions croissantes de la presse internationales, des réactions d'autres établissements d'enseignement supérieur et de l'impossibilité pour le gouvernement de destituer directement le recteur. Au moins sept établissements de différents États du pays manifestent contre la démission; de même, des étudiant-e-s - majoritairement mexicains - organisent des manifestations dans onze pays. Cette situation renforce la survie du mouvement et «l'esprit de triomphe » du CNH et des « masses en lutte » (Martínez Della Rocca 2010 : 356-357) et contrecarre provisoirement les effets de la démobilisation.

24. On le remarque en particulier avec le courrier du 18 septembre du ministre de l'Intérieur, Luis Echeverría Alvarez, dans lequel il est proposé une première réunion de négociation le matin du 2 octobre. Le 18 septembre, alors que le dialogue est planifié, la cité universitaire, puis l'IPN sont occupés militairement; le 2 octobre, dans l'après-midi, a lieu le massacre de Tlatelolco. 
25. Témoignage de Raúl Moreno Wonchee in González Marín 2003 : 73.

26. Témoignage de Luis Tomás Cervantes Cabeza de Vaca in González Marín 2003 : 100-101.

27. Témoignage de Marcelino Perelló in Echevarría 2008, $5^{\text {ème }}$ partie, 0 :01-1:48.

28. Témoignage de Margarita Suzán in Echevarría 2008, $5^{\text {ème }}$ partie, $6: 58-7: 37$.

29. Témoignage de Luis Tomás Cervantes Cabeza de Vaca in Varios Autores $2007:$ 80-81.

\section{RÉSUMÉS}

Cet article est consacré à l'analyse de la mobilisation et démobilisation dans le cas du mouvement étudiant de 1968 dans la ville de Mexico. En partant d'un rappel du contexte où s'enracine la lutte et en suivant les échanges de coups entre protagonistes durant les sept mois du conflit, il s'intéresse au rôle de la répression dans le processus de mobilisation et démobilisation. En étudiant, d'une part, la dynamique interne du mouvement en lien avec la configuration sociopolitique qui la rend possible ou la limite et, d'autre part, des témoignages d'activistes afin de cerner la perception des coûts et des risques de la mobilisation, cet article souligne que le poids de la répression dans le cycle de protestation prend sens quand la répression est liée aux risques de la participation et à sa dimension émotionnelle dans une configuration spécifique.

This article analyses the process of mobilization and demobilization in the case of the 1968 student movement in Mexico City. It begins with a reminder of the context in which the struggle is rooted, and goes on analyzing the fight between protagonists during seven months of conflict; it focuses on the role of repression in the process of mobilization and demobilization. By studying, on the one hand, the internal dynamics of the movement in relation to the sociopolitical configuration making possible or limiting this same dynamics, and, on the other, testimonies of activists to identify the perception of the mobilization's costs and risks, this article highlights that the weight of repression in the protest cycle makes sense if linked to the costs and risks of participation and to the emotional dimension of repression in a specific configuration.

\section{INDEX}

Mots-clés : Démobilisation, émotions, Mexique, mouvement étudiant, répression.

Keywords : Demobilization, emotions, Mexico, repression, student movement.

\section{AUTEURS}

\section{GUADALUPE OLIVIER-TÉLLEZ}

Universidad Pedagógica Nacional, Mexico

\section{SERGIO TAMAYO FLORES-ALATORRE}

Universidad Autónoma Metropolitana, unité Azcapotzalco, Mexico 
MICHAEL VOEGTLI

Université de Neuchâtel 